\title{
Host Modulation
}

\author{
Mohindra $\mathrm{K}^{*}$ and Nirola A \\ Department of Periodontics \& Oral Implantology, Laxmi \\ Bai Institute of Dental Sciences and Hospital, India \\ *Correspondling author: Kanika Mohindra, \\ Department of Periodontics \& Oral Implantology, Laxmi \\ Bai Institute of Dental Sciences and Hospital, 83 New Lal \\ Bagh, Patiala, India
}

Received: June 09, 2017; Accepted: June 30, 2017;

Published: July 12, 2017

\begin{abstract}
Although bacterial plaque is primary etiologic factor in the initiation and progression of periodontal diseases, the host's reactions to the presence of the bacteria are the one which mediates the periodontal tissue destruction. The periodontal tissue destruction is believed to be due to the host response, it is logical to consider therapeutic approaches that modulate the host response in addition to antibacterial and analgesic approaches in the management of periodontal diseases. A number of host modulatory agents like chemically modified tetracyclines (CMT), non-steroidal anti-inflammatory drugs, and bisphosphonates are effective in treating periodontal diseases in adjunct to the nonsurgical and surgical periodontal therapy. This paper reviews the Host Modulatory Therapy which is the effective means when used as an adjunct to mechanical periodontal therapy in treating periodontal diseases.
\end{abstract}

Keywords: Bisphosphonates; Chemically modified tetracyclines; Host modulation; Non-steroidal anti-inflammatory drugs; Periodontal disease

\section{Introduction}

Periodontitis is a health concern for centuries and are the most important causes of pain, discomfort, and tooth loss in adults. While a significant portion of the population is susceptible to periodontitis, there are those that are relatively resistant to the severe forms of periodontal disease. This leads to the hypothesis that, there are susceptibility factors or risk factors that modulate susceptibility or resistance of individuals to destructive periodontal disease.

The progression of periodontal disease is adversely influenced by a number of risk factors and risk indicators like diabetes, gender, age, hereditary and smoking. Once it was thought that the presence of pathogenic bacteria in the gingival sulcus was solely responsible for tissue destruction, traditional treatments focused on reducing the bacterial load through scaling and root planning, systemic and locally delivered antimicrobial drugs and antiseptics, however these treatment modalities were ineffective or less effective in all patients affected by periodontal disease.

Clinical evidence has demonstrated that not all individuals have the same response to similar amounts of plaque accumulation. There are patients with moderate and advanced disease who have very little plaque while other patients with little disease have large amounts of plaque. Most importantly, the plaque quantity, as well as the presence of specific bacteria, to the severity of periodontitis indicates that a substantial part of the variation in clinical severity of disease may be explained by factors other than the bacterial challenge. It should be emphasized that this statement in no way means that bacterial plaque is unimportant- in fact, it is quite the contrary.

Bacterial plaque is absolutely essential for the initiation and progression of periodontitis. However, it now appears that once the bacteria are present, the amount of periodontitis that a patient develops is due to factors related to the body's response to the bacterial challenge. It is in these disease susceptible individuals, the excessive host response leads to destruction of periodontal structures.
Many individuals harbor the putative pathogens associated with periodontal disease but they do not develop the disease. Current data suggests that plaque biofilm and associated host response are mainly involved in the pathogenesis of periodontitis. Certain putative periodontal pathogens predominantly Gram-negative, anaerobic bacteria within biofilm are associated with periodontal disease initiation and progression. The microbial challenge consisting of antigens, lipopolysaccharide and destruction is primarily by the host responses. The host responses are of mainly two types: antiinflammatory or protective and proinflammatory or destructive $[1,2]$.

\section{Mechanism of host induced periodontal destruction}

Concepts of the etiology of periodontal disease have changed markedly in the last four decades. In 1985 research began focus to on bacterial- host interactions. Several specific subgingival oral bacteria including porphyromonus gingivalis, aggregatibacter actinomycetemcomitans, prevotela intermedia, bacteroides forsythus and perhaps others such as campylobacter rectus, fusobacterium nucleatum, and spirochetes are associated with severe forms of periodontal disease [3]. The microbial challenge consisting of antigens, lipopolysaccharide (LPS), and other virulence factors stimulates host responses which result in disease limited to the gingiva (i.e., gingivitis) or initiation of periodontitis.

Protective aspects of host response include recruitment of neutrophils, production of protective antibodies and possibly the release of anti-inflammatory cytokines including transforming growth factor (TGF- $\beta$ ), interleukin-4 (IL-4), IL-10, and IL-12. Perpetuation of the host response due to a persistent bacterial challenge disrupts homeostatic mechanisms and results in release of mediators including proinflammatory cytokines (e.g., IL-1, IL-6, tumor necrosis factor(TNF- $\alpha$ ), proteases (e.g., Matrix Metalloproteinases), and prostanoids (e.g., prostaglandin E2 [PGE2]), which can promote extracellular matrix destruction in the gingiva and stimulate bone resorption.

The production of collagenase from infiltrating neutrophils and resident periodontal tissue cells is part of the natural host response 
to infection, in periodontal disease and other chronic inflammatory diseases. There is an imbalance between the level of activated tissue destroying matrix metallo proteinases (MMPs) and their endogenous inhibitors. These cascades of events from inflammation collectively lead to gingival recession, pocket formation, tooth mobility and tooth loss. It is clear then that host factors play a major role in the pathogenesis of periodontal disease [4].

\section{Host response modulation}

The concept of host modulation has been universally implemented since last few decades by the physicians in treatment of chronic diseases such as rheumatoid arthritis and osteoporosis. Although, Paul Goldhaber and Max Goodson began to implicate arachidonic acid metabolites as important inflammatory mediators of the bone loss in periodontitis in the 1970s but the concept of host modulation in dentistry was introduced by William and Golub in 1990 [5]. They concluded that "There are compelling data from animal and human trials indicating that pharmacologic agents that modulate the host responses believed to be involved in the pathogenesis of periodontal destruction may be effective in slowing progression of periodontal disease" [6].

In 1992, Golub and colleagues discussed host modulation with tetracyclines and their chemically modified analogues [7]. After introduction of this concept by William (1990), various host modulation agents have been developed and are currently being investigated, examples of these agents are NSAIDs, tetracyclines, chemically modified tetracycline, anticytokines agents(IL-1/TNF blockers), recombinant human IL-11, recombinant tissue inhibitor of matrix metalloproteinase, synthetic matrix metalloproteinase inhibitors and bisphosphonates $[8,9]$.

However, currently only one systemically administered agent, subantimicrobial dosage of doxycycline (SDD), has been approved by the United States Food and Drug Administration to be used for host modulation in periodontal disease [10].

The aim of host modulation therapy (HMT) is to reduce the tissue damage and stabilize or even regenerate the periodontium by modifying or down regulating destructive aspects of host response and up regulating protective or regenerative responses. The host immune and inflammatory response is primarily responsible for the periodontal tissue destruction. Various HMT developed block the specific pathways responsible for the destruction of periodontal tissue [11].

Host modulation therapeutics: Modulation is the alteration of function or status of something in response to a stimulus or an altered chemical or physical environment. In periodontics the concept of host modulation therapy is to reduce tissue destruction and stabilize or even regenerate the periodontium by modifying or down regulating destructive aspects of host response and up regulating protective or regenerative responses. As discussed earlier in pathogenesis of periodontal disease the prime destructive agents are MMPs, cytokines, arachidonic acid metabolites which result in increased probing depths (PD), clinical attachment loss (CAL), bleeding on probing (BOP) and alteration in levels of bio-chemical markers $[12,13]$. Therefore, the current host modulation therapies focus on reductions of these destructive enzymes by either systemic or local administration of host modulating agents $[14,15]$.

\section{Classification of host modulatory agents}

The host modulatory agents can be broadly classified as

- Agents preventing destruction (those which down regulate the destructive aspects of host response)

- Agents promoting resolution \& healing ( those which upregulate the protective or regenerative responses)

\section{Agents Preventing Destruction}

\section{Matrix metalloproteinase inhibitors}

Matrix Metalloproteinases (MMP) are zinc and calcium dependent endopeptidases secreted by polymorphonuclearneutrophils, macrophages, fibroblasts, epithelial cells, osteoblasts and osteoclasts. MMP's destroy extracellular components like collagen, gelatin, laminin, fibronectin and proteoglycans [16]. The activity of MMP's increases in chronic inflammatory conditions like periodontitis, rheumatoid arthritis. MMP's inhibitors act by inhibiting the release or synthesis of these enzymes by blocking the activation of precursor forms of these MMP's (pro- MMPs) [16]. This pro-MMP inhibits the activity of mature MMP's and stimulates the synthesis of endogenous tissue inhibitors of MMP's thereby protecting the host's endogenous inhibitors from proteolytic inactivation. The endogenous MMP inhibitors are TIMP (tissue inhibitor of metalloproteinase) and alpha-2 macroglobulin.

Exogenous inhibitors are present that includes zinc and calcium chelating agents, phosphorous containing peptides, sulfur based inhibitors, hydroxamic acid inhibitors. They are commercially available as; GalardinTM, BatimastatTM, MarimastatTM. The enzyme inhibitory spectrum of MarimastatTM and BatimastatTM reveals that it is capable of inhibiting MMP 1,2,3,7 \& 9 [17].

- Tetracycline, synthetic tetracyclines and chemically modified tetracyclines (CMTs): Tetracycline is a broad spectrum antibiotic prescribed for many systemic and periodontal infections. Due to its high concentration and secretion in GCF they provide effective antimicrobial cover locally for periodontal pathogens on non-vascular wall of periodontal pocket. In addition to its antibacterial property, tetracyclines also possess anti-collagenases effect due to inhibition of MMPs activity [18]. MMPs are Zinc and Calcium dependant endopeptidases. Tetracycline causes chelation of these ions thereby directly inhibiting the tissue degradation by these enzymes. Direct inhibition of MMPs and anti-inflammatory effect by blockade of hypochlorous acid, both these effects of tetracyclines also prevent the activation of latent pro- MMPs, therefore, further reducing the tissue destruction.

Tetracyclines also inhibit osteoclast and osteoblast derived MMPs thus lead to reduction of alveolar bone resorption $[19,20]$. Minocycline and doxycycline are synthetic tetracyclines, both these drugs have the characteristics to inhibit collagenase enzymes [21].

Doxycycline is also more effective in blocking PMN type collagenases (MMP-8) than fibroblast type collagenases (MMP-1), thus it does not interfere with the normal tissue turnover.

- CMTs are developed by either addition or deletion of functional groups in the central structure of tetracyclines. Currently, there are about ten CMTs developed and under clinical trials mainly; CMT-1 (4 dedimethylaminotetracycline), CMT -2 (tetracyclino- 
nitrile), CMT-3 (6-deoxy-6-demethyl-4-de-dimethylaminotetracycline), CMT-4 (7-chloro-4-de-dimethylamino-tetracycline), CMT-5 (tetracycline pyrazole), CMT-6 (4-dedimethyl amino 4-hydroxytetracycline), CMT-7 (12-deoxy-4-dedimethyl aminotetracycline) and CMT-8 (4-dedimethylaminodoxycycline). CMTs cause inhibition of MMPs activity, reduction of proinflammatory mediators, scavenges reactive oxygen species (ROS) and reactive nitrogen species [22]. CMTs in contrast to conventional tetracyclines do not cause gastrointestinal upsets and its therapeutic effects can be achieved with less frequent administration.

- Sub-antimicrobial dose doxycycline (SDD): Though numerous MMP inhibitors have been investigated, only tetracycline based host modulating agent, i.e. SDD- subantimicrobial dose of doxycycline (Doxycycline hyclate $20 \mathrm{mg}$; Periostat, CollaGenex, Pharmaceuticals Newton PA) has been approved by Food and drug administration (FDA) to be used as an adjunct to periodontal treatment [23]. A typical prescription for Periostat $(20 \mathrm{mg}$ doxycycline tablets) is for at least 3 months (180 tablets, 1 tablet twice a day until complete), and refills may be provided for longer courses of therapy [24]. Doxycycline has the ability to down regulate matrix metalloproteinases (MMPs), a family of zinc-dependant enzymes that are capable of degrading extracellular matrix molecules, including collagen.

\section{Non-steroidal anti inflammatory drugs (NSAIDs)}

Elevated PGE2 levels [25] and other amino acid (AA) metabolites have been reported in GCF and have been associated with destruction of periodontal tissue [26]. Therefore, AA metabolites reduction has been focused since decades to prevent the progression of periodontal disease. NSAIDs inhibit the enzyme cyclooxygenase (CO) and lipooxygenase (LO), thereby inhibit production of prostaglandins, prostacyclines, thromboxanes, leukotrienes and other hydroxyeicosatetraenoic acids by these pathways. Reduced synthesis of prostaglandin causes decrease in bone resorption in experimental models.

Systemic NSAIDs e.g. indomethacin, naproxen, piroxicam, ibuprofen and flurbiprofen have been extensively studied for their role in inhibiting alveolar bone resorption and retarding the progression of periodontal disease [27-32]. However, on cessation of therapy, immediate recurrence of disease occurred thereby requiring its prolonged administration to sustain its therapeutic effects.

Topical NSAIDs such as piroxicam [30], ketoprofen [33], flurbiprofen [34] ibuprofen [35], meclofenamic acid [35] administration in the form of cream, gel or dentifrice use have also been advocated due to its lipophilic nature and their quick absorption into periodontal tissue to cause its therapeutic effect with greater efficacy at lower doses and with fewer adverse effects [36].

Prolonged administration of NSAIDs is cautioned due to its adverse effects including gastrointestinal upset, renal, hepatic and hemorrhage impairment due to nonselective inhibition of COX-1 and COX-2 enzymes $[37,38]$. Therefore current clinical trials focus on the use of selective NSAIDs (COX-2) inhibitors, as these are associated with similar clinical effects with fewer gastrointestinal adverse effects as compared to nonselective NSAIDs $[27,39,40]$. Currently, FDA has not approved any NSAID formulation for host modulation therapy in periodontics and future research is required to determine its suitability as a host modulating agent.

Triclosan (2,4,4-trichloro 2-hydroxydiphenyl ether) possesses non-ionic antimicrobial activity and anti-inflammatory property by inhibiting CO and LO pathways [41]. Due to this dual activity, triclosan provides an effective remedy to prevent attachment loss in periodontitis. Triclosan/copolymer dentifrices are available inmarket indicated for patients susceptible to periodontitis by reducing plaque and calculus deposits and resolving gingivitis [42].

\section{Bone sparing agents (Bisphosphonates)}

Bisphosphonates are analogs of pyrophosphate having high affinity for calcium phosphate in bone tissue [43]. Bisphosphonate (e.g. Alendronate) inhibit osteoclasts activity and posses property of inhibiting ions dependant enzyme activity (MMPs) through chelation of cations $[44,45]$. These agents inhibit the loss of bone density and prevent normal bone turnover. At cellular level, they inhibit osteoclast recruitment and adhesion, increase osteoblast number by differentiation and decrease release of cytokines by macrophages/ neutrophils.

Few clinical studies have been performed to determine bisphosphonate usage for treatment of periodontitis as an adjunct with SRP. These studies have shown significant [46] and modest $[47,48]$ improvements in bone levels when evaluated by clinical and radiographic methods. One larger 12 months study on 70 subjects demonstrated modest clinical improvement but no improvements in radiographic bone mass after bisphosphonate usage [49]. Despite conflicting results in bone levels in various clinical studies, bisphosphonate therapy shows improvement in CAL, PD and BOP. Its prolonged usage in periodontics is cautioned due to its adverse effects such as osteonecrosis of the jaws (ONJ); however, the incidence of $\mathrm{ONJ}$ is much lesser with oral bisphosphonate therapy as compared to high dose intravenous bisphosphonate therapies. Currently, FDA has approved bisphosphonates for systemic bone loss only, therefore, more extensive clinical research is required for its indication as adjunct host modulating agent in periodontics [50].

\section{Cytokines (immune) modulation}

As discussed previously in pathogenesis of periodontal disease host cell derived proinflammatory cytokines and anti-inflammatory cytokines production determines the extent of destruction of connective tissue and alveolar bone. Immune modulation therapy aims at reduction of proinflammatory cytokines and/or increasing anti-inflammatory cytokines level. The use of cytokine receptor antagonist (IL-1/TNFblocker) have been investigated and showed reduction in periodontal disease progression in experimental models. Anti-inflammatory cytokines (IL-4 and IL-10) inhibits the release of IL-1, TNF- $\alpha$, nitrous oxide (NO) and other destructive molecules by the host cells. IL-4 is also responsible for increasing the number of IL-1 receptor antagonist thereby decreasing the tissue destruction [52]. The exogenous administrations of IL- 4 and recombinant IL11 in experimental models have been associated with reduction in disease progression e.g. in Arthritis, Periodontitis etc [51,52].

\section{RANK/RANKL/Osteoprotegerin Rank (Receptor activator of nuclear factor $K \beta$ )/RANKL}

Receptor activator for nuclear factor $\kappa \beta$ ligand interaction is 
responsible for differentiation \& maturation of osteoclast precursor cells to activate osteoclast. Osteoprotegerin (OPG) acts as a decoy receptor expressed by osteoblastic cells which binds to RANKL \& inhibits osteoclast development. OPG prevents the binding of RANKL to specific membrane bound receptors expressed in osteoclast precursor cells. Expression of the RANKL gene in osteoblasts/stromal cells is enhanced by Vitamin D3, PTH, IL-1, IL-6, IL- 17, TNF- $\alpha$, BMP, PGE2. Denosumab, a fully human monoclonal antibody targets RANKL and this could be a potential host modulatory therapy [53]. OPG is the natural inhibitor of RANKL, produced by human PDL cells, gingival fibroblasts and epithelial cells. OPG as a therapeutic agent was described by Simon, et al. 1997 [54]. Interference with the RANK-RANKL-OPG axis had a protective effect on osteoclastogenesis and alveolar bone loss in animal studies.

\section{Hormone replacement therapy}

It refers to any form of hormone therapy where in the patient, in the course of treatment receives hormones either to supplement a lack of naturally occurring hormones. Use of hormone replacement therapy in the management of rheumatoid arthritis has produced conflicting results. Hormone replacement therapy in post menopausal women shows a slight improvement in periodontal condition but generally such improvements appear to be small and of debatable value. Potentially large number of significant side effects is present. Although this strategy has been not recommended for proposed management of periodontitis [55].

\section{Cathepsin K inhibitors}

Cathepsin $\mathrm{K}$ is a cysteine proteinase of the papain super family. It is selectively expressed in osteoclasts and plays pivotal role in degradation of bone matrix [56]. It is the only known mammalian proteinase that can solubilize both, type I and type II collagens increased in gingival crevicular fluid in patients with periodontitis, which correlated with an increased concentration of RANKL. Cathepsin $\mathrm{K}$ has been viewed as an attractive target for modulating bone resorption. Stroup, et al. in 2001 [57] stated that cathepsin K inhibitors causes inhibition of human cathepsin $\mathrm{k}$ which in turn induces a reduction in bone in non human primates whereas Deal, et al. in 2009 [58] states that a number of prototype cathepsin inhibitors have now entered clinical trials for the management of osteoporosis. However none have yet been made available for use as host modulation therapy for periodontitis.

\section{Vitamin D}

Vitamin D is crucial for a wide variety of organ systems; nevertheless, its deficiency is highly prevalent, present in $30-50 \%$ of the general population. Evidence has demonstrated that vitamin $\mathrm{D}$ deficiency may place subjects at risk for not only low mineral bone density/osteoporosis and osteopenia, but also infectious and chronic inflammatory diseases. Due its effect on bone and mineral metabolism, innate immunity, and several vitamin $\mathrm{D}$ receptor gene polymorphisms, vitamin $\mathrm{D}$ has been reported to be associated with the periodontal disease. More recent studies showed significant associations between periodontal health and intake of vitamin $\mathrm{D}$ and calcium, and that dietary supplementation with calcium and vitamin D may improve periodontal health, increase bone mineral density in the mandible and inhibit alveolar bone resorption. In a recently published longitudinal study, Garcia, et al. reported that calcium and vitamin D supplementation may reduce the severity of periodontal disease if used at doses higher than 800-1,000 IU daily 28. They also noted that vitamin $\mathrm{D}$, in addition to its role in bone and calcium homeostasis, acts as an anti-inflammatory agent because it inhibits immune cell cytokine expression and causes monocyte/macrophages to secrete molecules that have a strong antibiotic effect [59].

\section{Statins}

Statins, 3-hydroxy-3-methylglutarylcoenzyme A (HMG CoA) reductase inhibitors, can be fermentation derived statins include simvastatin, pravastatin whereas atorvastatin, cerivastatin, fluvastatin, pitavastatin and rosuvastatin are synthetic stains. Synthetic statins have a higher potency as compared to the fermented statins. Also statins influence the production of receptor activator of nuclear factor kappa-B ligand (RANKL) and osteoprotegrin by human gingival fibroblasts to favour bone catabolism under non-inflammatory conditions. Clinical trial on patients with chronic periodontitis showed that there was a greater decrease in gingival index (GI) and probing depth (PD) and more clinical attachment level gain with significant intrabony defect fills at sites treated with scaling and root planing (SRP) plus locally delivered simvastatin than with SRP alone. Beneficial effect of atorvastatin on bone alveolar loss and tooth mobility in subjects with periodontal disease has also been identified [60].

\section{Wnt pathway}

Wnt canonical pathway and the transcription factor activator protein-1 are important for the regulation of osteoprotegerin production in osteoblasts. Within this process betacatenin plays an important role in the Wnt signaling pathway and bone remodeling. Beta catenin could enhance IL $-1 \alpha$ induced OPG production. Wnt pathway has an important link between inflammation and bone metabolism and has novel target for treating bone erosive conditions. DICKKOPF-1(DKK- 1), a glycoprotein that can inhibit the Wnt pathway. Pinzone, et al. in 2009, states that use of a DKK1, led to an increase in the amount of trabecular bone, an increased number of osteoblasts and increased osteocalcin levels $[61,62]$.

\section{Protease activated receptor $\mathbf{2}$ agonists \& antagonists}

Protease activated receptor 2 (PAR2) also known as coagulation factor II (thrombin) receptor-like 1 (F2RL1) or G-protein coupled receptor 11 (GPR11) is a protein that in humans is encoded by the F2RL1 gene. PAR2 modulates inflammatory responses and acts as a sensor for proteolytic enzymes generated during infection. Smith, et al. in 2004 [63], states that PAR 2 activation inhibits bone resorption by inhibiting osteoclasts differentiation.

\section{Histone deacetylase inhibitors}

This act by inhibiting angiogenesis, causing a protective effect on bone thereby inhibits the production of proinflammatory cytokines. Lin, et al. in 2007 [64] described the action of Histone deacetylase inhibitors in animal models of rheumatoid arthritis where there is decrease in bone destruction. However this strategy has not been applied for the management of periodontitis.

\section{Nitric oxide synthase inhibitors}

Nitric oxide (NO) is not only important in host defense and homeostasis but it is also regarded as harmful and has been 
implicated in the pathogenesis of a wide variety of inflammatory and autoimmune diseases.

Nitric oxide may activate proinflammatory enzymes such as cyclooxygenase and metalloproteinases, which, in turn, may also contribute to periodontal tissue damage. The most commonly used drug is mercapto ethyl guanidine which blocks inducible Nitric oxide synthase (iNOS), which in turn inhibits COX and scavenges peroxynitrate. Lohinai, et al. 1998-found a reduction of alveolar bone loss and gingival inflammation after the use of a selective iNOS inhibitor- mercaptoethylguanidine [65].

\section{Antagonists for adhesion molecules}

Studies have proven that there are increasing levels of ICAM1(Intercellular Adhesion molecule-1), LECAM-1(Neutrophil Lectin Adhesion molecule-1) and PECAM- 1(Platelet/Endothelial cell adhesion molecule-1) expression with increasing degrees of inflammation in cases of both gingivitis and periodontitis [66] ICAM-1 \& E- selectin inhibitors

- Tepoxalin

- Sodium Cromoglycate,

- BMS-190394

\section{Cell signalling pathway destruction}

As soon as the bacterial biofilm accumulates in the gingival margin, an inflammatory process is initiated, triggering a dynamic cascade of events. The main purpose of these events is the combat of microbial invades through pro-inflammatory actions. This proinflammatory action depends on the recognition of external antigenic stimuli by host leukocyte of the innate immune response.

This recognition of the external stimuli triggers a signal that travels through cytoplasm and then reaches nucleus altering the pattern of gene expression. In a periodontal disease, the most important pathways includes the NF- $\kappa \beta$, p38, ERK, JNK pathways [67]. The cell signaling pathway can be descripted by;

- Nuclear factor-kappa B (NF-kB) inhibitors

- $\quad$ (IKK-B) inhibitors (Inhibitor of kappa-B kinase)

- $\quad$ Extracellular signal-regulated kinases (ERK) 1/2inhibitors

- Jun N-terminal kinases (JNK) inhibitors

- $\quad$ p-38 inhibitors

- NF- $\mathrm{kB}$ Inhibitors- Proteasome inhibitors block NF- $\mathrm{KB}$ activation.

- $\quad$ IKK- $\beta$ inhibition-IKK- $\beta$ is activated following the binding of RANK to RANKL. And this inhibition is a target for the inactivation of nuclear factor - kappa B. BMS -345541 - first IKK inhibitor [68].

\section{Modulation of toll like receptors (TLR)}

Toll-like receptors (TLRs) are a class of proteins that play a key role in the innate immune system. They are single, membrane spanning, non-catalytic receptors usually expressed in sentinel cells such as macrophages and dendritic cells, that recognize structurally conserved molecules derived from microbes. Strategies for inhibition of Toll like Receptors function (TLR) and signaling includes;
- Natural or synthetic agonists blocks ligand binding

- Monoclonal antibodies blocks ligand binding

- Soluble decoy toll like receptors inhibits TLR or co-receptor interactions

- Kinase inhibitors bring about a negative regulation of toll like receptors downstream signalling.

- Anticytokine agents neutralize toll like receptors thereby induce proinflammatory cytokines [69].

\section{Periodontal vaccines}

Vaccination is a process that induces specific immune resistance to a bacterial or viral infection. In the early twentieth century, three periodontal vaccines were employed, which includes;

- Pure cultures of streptococcus and other organisms

- Autogenous vaccines

- $\quad$ Stock vaccines [70].

Example includes Vancott's vaccine and Inava endocarp vaccine

There are 3 types of periodontal immunization. This includes;

Active immunization

${ }^{*}$ Whole bacterial cells

${ }^{*}$ Sub unit vaccines

Synthetic peptides as antigens

Passive immunization

${ }^{\star}$ Murine monoclonal antibody

*Plantibodies

Genetic immunization

${ }^{\star}$ Plasmid vaccines

${ }^{\star}$ Live, viral vector vaccines.

Antigens used for active immunization includes:

- Bacterial whole cells

- P. gingivalisfimbriae

-P. gingivaliscysteine protease [71]

\section{Agents Promoting Resolution and Healing}

\section{Pro resolution mediators}

Resolution is an active process involving biochemical circuits that actively biosynthesize local mediators within the resolution phase target resolution rather than inhibiting inflammation by the role of stromal cells where there is withdrawal of survival signals, normalization of chemokine gradients, induction of resolution programs that allow infiltrating cells to undergo apoptosis or exit the inflamed tissue through draining lymphatics. Anti inflammatory \& pro resolving molecules:

- $\quad$ Lipoxins (LX) 
- $\quad$ Resolvins of the E Series (RvE)

- $\quad$ Resolvins of the D Series (RvD)

- $\quad$ Aspirin Triggered Epimeric Forms from DHA

- The Neuroprotectins/Protectins

- $\quad$ Aspirin Triggered Lipoxins (ATL)

- Glucocorticoid induced annexin-1

- Melanocortins /nuclear receptor agonists

- $\quad$ Hemeoxygenase 1 .

Lipoxins: Lipoxins are members of the Eicosanoid family produced by lipoxygenase mediated metabolism of Arachidonic Acid (AA). They are generated by cell to cell interactions (transcellular biosynthesis). Crevicular fluid samples from localized aggressive periodontitis (LAP) patients were examined and found to contain PGE2 (prostaglandins E2), LTB4 (leukotriene B4), and LXA4 (lipoxinA4). Neutrophils from peripheral blood of LAP patients, but not from healthy volunteers, also generated LXA4, suggesting that it may also have a role in periodontal diseases [72].

Resolvins: Lipid mediators that are induced in their resolution phase following inflammation. Resolvins are synthesized from omega 3 PUFA (Poly unsaturated fatty acid)- EPA (Eicosapantaenoic acid) \& DHA (Docosahexaenoic acid). They demonstrate potent anti inflammatory and immunomodulatory actions in the nanogram dose range in vivo. RvE1 (ResolvinE1) Topical application of RvE1 in experimental model of periodontitis in rabbits prevented the progression of periodontitis. There was no neutrophils and tissue damage and lack of osteoclast proliferation. A radiographic evidence of decreased percentage of bone loss is also seen.

Protectins: Endogenous DHA is converted in to another family of lipid mediators called as protectins, that is owing to its potent protective activity in inflammatory and neural system, known as protectinD1 [73]. Protectin D1 is also known as neuroprotectin D1, when produced by neural tissues [74]. However, none of these proresolution strategies have been approved for the management of periodontal disease.

Enamel matrix derivatives: Enamel matrix derivatives are primarily composed of amelogenin which can promote periodontal ligament fibroblasts proliferation and growth. The only local host modulation agent currently approved by the FDA for adjunctive use during surgery is EmdogainTM. Emdogain contains proteins that are believed to regenerate tooth attachment. It has the capacity to regenerate about $1 \mathrm{~mm}$ more tissues than surgical debridement alone [75].

Growth and differentiation factors: Growth differentiation factors (GDFs) are a subfamily of proteins belonging to the transforming growth factor $\beta$ superfamily that have functions predominantly in development [76].

Platelet derived growth factor plays a significant role in angiogenesis. It is a element In cellular division for fibroblast. Insulin like growth factors are proteins that resemble insulin. Bone morphogenetic proteins are group of growth factors also known as cytokines and metabologens. BMP-2 \& BMP-7 are involved in promoting resolution \& healing. Among the other bone morphogenetic proteins, BMP-2 \& BMP-7 belong to Transforming Growth Factor- $\beta$. BMP-2 \& BMP-7 plays a key role in osteoblast differentiation [77].

\section{Newer directions in host modulation}

Current understanding of periodontal disease initiation states that the presence of pathogens in the periodontal pocket leads to pocket formation with further tissue destruction. Van Dyke (2007) [78] propose that by introducing "resolving agents", perhaps the resolution of inflammation leads to disappearance of the pathogenic bacteria by removal of their food source. If this hypothesis is proven correct by further research, it would indeed lead us to rethink our clinical treatment strategies. By moving towards drugs that promote resolution, rather than just anti-inflammation, we may be able to harness the advantages in the inflammatory cascade, while leading to a speedy return to homeostasis, and health. The use of some of these pro-resolution agents has already been tested in animal models [79], where the use of RvE1 has been shown to markedly reduce periodontal inflammation, with regeneration of bone to pre-ligature height, and regeneration of cementum and an organized periodontal ligament.

Bannenberg, et al. (2005) [80] have proposed a set of resolution indices in an attempt to set benchmarks to assess the impact of proresolving agents. It is possible that these indices might introduce new perspectives to the field of drug research.

Needless to say, the optimum drug regimen would have to be developed after delineating the exact mechanisms between acute and chronic inflammatory processes. This, in turn, might potentially translate into arresting periodontal disease before it becomes a chronic process. If pro-resolving agents continue to be developed, then a significant clinical implication would be a change in how we differentiate between the clinical signs of inflammation or resolution, and use this information in subsequent therapy. We are traditionally attuned to identifying inflammation solely by its presence or absence, using bleeding on probing as a clinical parameter. The introduction of a new "resolution" parameter that does not solely depend on the absence of inflammation might add an interesting concept to periodontal disease diagnosis and treatment. Development of drugs based on endogenous mediators inherent to resolution (Gilroy, et al. 2004) [81] may represent a new strategy in anti-inflammatory treatment in general and periodontal therapy in particular. However, with the exception of some drugs that target apoptosis (Murphy, et al. 2003) [82], and drugs like methotraxate, sulphasalazine, and FK506 (Hasko \& Cronstein 2004) [83] that target adenosine, we are not aware of many commercially available drugs that utilize the resolution pathway. As more randomized clinical trials examining resolving agents become available, we will continue to move towards a commercially available 'pro-resolution' drug for periodontal disease.

\section{Conclusion}

The improved understanding of the host-bacterial interactions and the host immuno-inflammatory response leading to periodontal tissue destruction has led to the development of HMT. Though the efficacy and usefulness of host modulating agents have been 
demonstrated by many clinical trials and have been approved by FDA for the management of periodontitis, yet the risk/benefit ratio relating to the use of these drugs has yet to be established. Multicenter clinical trials are necessary to fully evaluate the benefits of these agents and to weigh their usefulness against the risks associated with their long-term administration. Furthermore, continuous research in this field would also enable fabrication of individualized treatment for periodontal disease targeting inflammatory host response. The current article emphasizes the promising potential of various host-modulating agents (the most crucial component of perioceutics) in the management of periodontal diseases.

\section{References}

1. Giannobile WV. Host-response therapeutics for periodontal diseases. J Periodontol. 2008; 79: 1592-1600.

2. Preshaw PM, Seymour RA, Heasman PA. Current concepts in periodontal pathogenesis. Dent Update. 2004; 31: 570-572, 574-578.

3. Genco R, Kornman K, Williams R, Offenbacher S, Zambon J, Ishikawa I, et al. Consensus report periodontal diseases: pathogenesis and microbial factors. Ann Periodontol. 1996; 1: 926-932.

4. Yetkin Ay Z, Sütçü R, Uskun E, Bozkurt F, Berker E. The impact of the IL11: IL17 ratio on the chronic periodontitis pathogenesis: a preliminary report. Oral Dis. 2009; 15: 93-99.

5. Preshaw PM. Host response modulation in periodontics. Periodontol 2000. 2008; 48: 92-110.

6. Williams RC. Periodontal disease. N Engl J Med. 1990; 322: 373-382.

7. Golub LM, Suomalainen K, Sorsa T. Host modulation with tetracyclines and their chemically modified analogues. Curr Opin Dent. 1992; 2: 80-90.

8. Giannobile WV. Host-response therapeutics for periodontal diseases. $J$ Periodontol. 2008; 79: 1592-1600.

9. Howell TH, Williams RC. Nonsteroidal antiinflammatory drugs as inhibitors of periodontal disease progression. Crit Rev Oral Biol Med. 1993; 4: 177-196

10. Williams RC. Periodontal disease. N Engl J Med. 1990; 322: 373-382.

11. Offenbacher S, Odle BM, Van Dyke TE. The use of crevicular fluid prostaglandin E2 levels as a predictor of periodontal attachment loss. $J$ Periodontal Res. 1986; 21: 101-112.

12. Giannobile WV. Host-response therapeutics for periodontal diseases. Periodontol. 2008; 79: 1592-1600.

13. Zahavi T, Caton JG. Subantimicrobial dose doxycycline-host modulation in the treatment of periodontitis. Oral Health. 2005; 36-47.

14. Oringer RJ; Research, Science, and Therapy Committee of the American Academy of Periodontology. Modulation of the host response in periodontal therapy. J Periodontol. 2002; 73: 460-470.

15. Reddy MS, Geurs NC, Gunsolley JC. Periodontal host modulation with antiproteinase, anti-inflammatory, and bonesparing agents: a systemic review. Ann Periodontol. 2003; 74: 12-37.

16. Tumuluri $\mathrm{V}$. Matrix Metalloproteinase regulation in periodontal treatment. $\mathrm{J}$ Periodontol. 2001; 22: 50-57.

17. Ryan ME, Ramamurthy S, Golub LM. Matrix metalloproteinases and their inhibition in periodontal treatment. Curr Opin Periodontol. 1996; 3: 85-96.

18. Sahitya S, Nugala B, Santosh Kumar BB. Matrix metalloproteinases. J Orofac Sci. 2010; 2: 75-81

19. Preshaw PM. Host response modulation in periodontics. Periodontol 2000 2008; 48: 92-110.

20. Golub LM, Lee HM, Ryan ME, Giannobile WV, Payne J, Sorsa T. Tetracyclines inhibit connective tissue breakdown by multiple nonantimicrobial mechanisms. Adv Dent Res. 1998: 12: 12-26.
21. Preshaw PM, Hefti AF, Jepsen S, Etienne D, Walker C, Bradshaw MH Subantimicrobial dose doxycycline as adjunctive treatment for periodontitis. A review. J Clin Periodontol. 2004; 31: 697-707.

22. Agnihotri R, Gaur S. Chemically modified tetracyclines: novel therapeutic agents in the management of chronic periodontitis. Indian J Pharmacol. 2012; 44: 161-167

23. Caton J, Ciancio S, Crout R, Hefti A, Polson A. Adjunctive use of subantimicrobial doxycycline therapy for periodontitis. J Dent Res. 1998; 77 : 1001.

24. Ryan ME. Nonsurgical approaches for the treatment of periodontal diseases. Dent Clin North Am. 2005; 49: 611-636, vii.

25. Noguchi K, Ishikawa I. The roles of cyclooxygenase-2 and prostaglandin E2 in periodontal disease. Periodontol. 2000. 2007; 43: 85-101.

26. Preshaw PM, Hefti AF, Jepsen S, Etienne D, Walker C, Bradshaw MH. Subantimicrobial dose doxycycline as adjunctive treatment for periodontitis. A review. J Clin Periodontol. 2004; 31: 697-707.

27. Nyman S, Schroeder HE, Lindhe J. Suppression of inflammation and bone resorption by indomethacin during experimental periodontitis in dogs. $J$ Periodontol. 1979; 50: 450-461.

28. Williams RC, Jeffcoat MK, Howell TH, Hall CM, Johnson HG, Wechter WJ, et al. Indomethacin or flurbiprofen treatment of periodontitis in beagles: comparison of effect on bone loss. J Periodontal Res. 1987; 22: 403-407.

29. Howell TH, Jeffcoat MK, Goldhaber P, Reddy MS, Kaplan ML, Johnson HG, et al. Inhibition of alveolar bone loss in beagles with the NSAID naproxen. $J$ Periodontal Res. 1991; 26: 498-501.

30. Howell T1, Fiorellini J, Weber HP, Williams RC. Effect of the NSAID piroxicam, topically administered, on the development of gingivitis in beagle dogs. J Periodontal Res. 1991; 26: 180-183.

31. Williams RC, Jeffcoat MK, Howell TH, Reddy MS, Johnson HG, Hall CM, et al. Ibuprofen: an inhibitor of alveolar bone resorption in beagles. J Periodontal Res. 1988; 23: 225-229.

32. Williams RC, Jeffcoat MK, Kaplan ML, Goldhaber P, Johnson HG, Wechter WJ. Flurbiprofen: a potent inhibitor of alveolar bone resorption in beagles. Science. 1985; 227: 640-642.

33. Paquette DW, Fiorellini JP, Martuscelli G, Oringer RJ, Howell TH, McCullough $\mathrm{JR}$, et al. Enantiospecific inhibition of ligature-induced periodontitis in beagles with topical (S)-ketoprofen. J Clin Periodontol. 1997; 24: 521-528.

34. Williams RC, Jeffcoat MK, Howell TH, Reddy MS, Johnson HG, Hall CM, et al. Topical flurbiprofen treatment of periodontitis in beagles. J Periodont Res. 1988; 23: 166-169.

35. Kornman KS, Blodgett RF, Brunsvold M, Holt SC. Effects of topical applications of meclofenamic acid and ibuprofen on bone loss, subgingival microbiota and gingival PMN response in the primate Macaca fascicularis. J Periodont Res. 1990; 25: 300-307

36. Jeffcoat MK, Reddy MS, Haigh S, Buchanan W, Doyle MJ, Meredith MP, et al. A comparison of topical ketorolac, systemic flurbiprofen, and placebo for the inhibition of bone loss in adult periodontitis. J Periodontol. 1995; 66: 329-338.

37. Velo GP, Milanino R. Nongastrointestinal adverse reactions to NSAID. J Rheumatol Suppl. 1990; 20: 42-45.

38. Hawkey CJ. Gastroduodenal problems associated with non-steroidal, antiinflammatory drugs (NSAIDs). Scand J Gastroenterol Suppl. 1993; 200: 9495.

39. Fitz Gerald GA, Patrono C. The coxibs, selective inhibitors of cyclooxygenase-2. N Engl J Med. 2001; 345: 433-442.

40. Greenwell H1, Bissada NF. Emerging concepts in periodontal therapy. Drugs 2002: 62: 2581-2587.

41. Gaffar A, Scherl D, Afflitto J, Coleman EJ. The effect of triclosan on mediators of gingival inflammation. J Clin Periodontol. 1995; 22: 480-484. 
42. Rosling B, Wannfors B, Volpe AR, Furuichi Y, Ramberg P, Lindhe J. The use of a triclosan/copolymer dentifrice may retard the progression of periodontitis. J Clin Periodontol. 1997; 24: 873-880.

43. Fleisch $\mathrm{H}$. Bisphosphonates. Pharmacology and use in the treatment of tumour-induced hypercalcaemic and metastatic bone disease. Drugs. 1991 42: 919-944.

44. Tenenbaum HC, Shelemay A, Girard B, Zohar R, Fritz PC. Bisphosphonates and periodontics: potential applications for regulation of bone mass in the periodontium and other therapeutic/diagnostic uses. J Periodontol. 2002; 73 : 813-822.

45. Nakaya H, Osawa G, Iwasaki N, Cochran DL, Kamoi K, Oates TW. Effects of bisphosphonate on matrix metalloproteinase enzymes in human periodontal ligament cells. J Periodontol. 2000; 71: 1158-1166.

46. Jeffcoat MK, Reddy MS. Alveolar bone loss and osteoporosis: evidence for a common mode of therapy using the bisphosphonate alendronate. In Davidovitch Z, Norton LA, editors. Biological mechanisms of tooth movement and craniofacial adaptation. Cambridge, MA: Harvard Society for the Advancement of Orthodontics. 1996; 365-373

47. Rocha M, Nava LE, Vazquez de la Torre C, Sanchez-Marin F, Garay-Sevilla $\mathrm{ME}$, Malacara JM. Clinical and radiological improvement of periodontal disease in patients with type 2 diabetes mellitus treated with alendronate: a randomized, placebo-controlled trial. J Periodontol. 2001; 72: 204-209.

48. Takaishi Y, Miki T, Nishizawa Y, Morii H. Clinical effect of etidronate on alveolar pyorrhoea associated with chronicmarginal periodontitis: report of four cases. J Int Med Res. 2001; 29: 355-365.

49. Lane N, Armitage GC, Loomer P. Bisphosphonate therapy improves the outcome of conventional periodontal treatment: results of a 12-month, randomized, placebo-controlled study. J Periodontol. 2005; 76: 1113-1122.

50. Palomo L, Liu J, Bissada NF. Skeletal bone diseases impact the periodontium: a review of bisphosphonate therapy. Expert Opin Pharmacother. 2007; 8 $309-315$.

51. Allen JB, Wong HL, Costa GL, Bienkowski M, Wahl SM. Suppression of monocyte function and differential regulation of IL-1 and IL-1ra by IL-4 contribute to resolution of experimental arthritis. J Immunol. 1993; 151: 4344 4351

52. Martuscelli G, Fiorellini JP, Crohin CC, Howell TH. The effect of interleukin-11 on the progression of ligature-induced periodontal disease in the beagle dog. J Periodontol. 2000; 71: 573-578.

53. Bartold PM, Cantley MD, Haynes DR. Mechanisms and control of pathologic bone loss in periodontitis. Periodontol. 2000. 2010; 53: 55-69.

54. Simonet WS, Lacey DL, Dunstan CR, Kelley M, Chang MS, Lüthy R, et al. Osteoprotegerin: a novel secreted protein involved in the regulation of bone density. Cell. 1997; 89: 309-319.

55. Simonet WS, Lacey DL, Dunstan CR, Kelley M, Chang MS, Lüthy R, Nguyen $H Q$, et al. Osteoprotegerin: a novel secreted protein involved in the regulation of bone density. Cell. 1997; 89: 309-319.

56. Pizzo G, Guigalia R, Locate ME. Effect of hormone replacement therapy periodontal status of menopausal women. Med Sci Monit. 2011; 17; 23-27.

57. Dennis S. Yamashita, Ward W. Smith, Baognang Zhao, Cheryl A. Janson, Thaddeus A. Tomaszek, Mary J. Bossard, et al. Structure and design of protein and selective cathepsin K inhibitors. J Am Chem Soc.1997; 119; $11351-11352$.

58. Stroup GB, Lark MW, Veber DF, Bhattacharrya A, Blake S, Dare LC, et al. Potent and selective inhibition of human cathepsin $\mathrm{K}$ leads to inhibition of bone resorption in-vivo in a nonhuman primate. J Bone Miner Res. 2001; 16 1739-1746.

59. Deal C. Future therapeutic targets in osteoporosis. Curr Opin Rheumatol. 2009; $21: 380-385$

60. M. Nathalia Garcia, Charles F. Hildebolt, D. Douglas Miley, Debra A. Dixon, Rex A. Couture, Roberto Civitelli, et al. One year effects of Vit $D$ and calcium supplements on chronic periodontitis. J Periodontol. 2011; 82: 25-32.
61. Grover HS, Luthra S, Maroo S, Maroo N. The pleotropic role of statins: Could it be the imminent host modulation agent in periodontics? Dent Res $\mathrm{J}$ (Isfahan). 2013; 10: 143-148.

62. Glass DA, Bialek P, Ahn JD, Starbuck M, Patel MS, Clevers H, et al. Canonica wnt signaling in differentiated osteoblast controls osteoclast differentiation. Dev Cell. 2005; 8: 751-764

63. Pinzone JJ, Hall BM, Thudi NK, Vonau M, Qiang YW, Rosol TJ, et al. The role of Dickkopf-1 in bone development, homeostasis, and disease. Blood. 2009; 113: 517-525.

64. Kawagoe J, Takizawa T, Matsumoto J, Tamiya M, Meek SE, Smith AJ, et al Effect of protease-activated receptor-2 deficiency on allergic dermatitis in the mouse ear. Jpn J Pharmacol. 2002; 88: 77-84

65. Lin HS, Hu CY, Chan HY, Liew YY, Huang HP, Baron, et al. Anti rheumatic activities of histonedeacetylase inhibitors in vivo in collagen induced arthritis in rodents. Br J Pharmacol. 2007; 150: 862-872.

66. Brennan PA, Thomas GJ, Langdon JD. The role of nitric oxide in ora diseases. Arch Oral Biol. 2003; 48: 93-100.

67. Page RC. The role of inflammatory mediators in the pathogenesis of periodontal disease. J Periodontal Res. 1991; 26: 230-242.

68. Ambili R, Santhi WS, Janam P. Expression of Activated transcription facto NF-?ß in periodontally diseased tissues. J Periodontol. 2005; 76: 1148-1153.

69. Schreiber S, Feagan B, DHaens G, Colombel JF, Geboes K, Yurcov M, et al. BIRB 796 study group. Oral p38 mitogenic-activated protein kinase inhibition with BIRB 796 for active Crohn's disease, a randomized, doubleblind placebo-controlled trial. Clin Gastroenterol Hepatol. 2006; 4: 325-334.

70. Zhang P, Liu J, Xu Q, Harber G, Feng X, Michalek SM, et al. TLR2-dependent modulation of osteoclastogenesis by Porphyromonas gingivalis through differential induction of NFATc1 and NF-kappaB. J Biol Chem. 2011; 286 24159-24169.

71. Seymour GJ, Gemmell E, Eastcoll J, Taubman MA. Immunopathogenesis of chronic inflammatory periodontal diseases: cellular \& molecular. J Periodontol Res. 1993; 28: 478-486.

72. Kudyar N, Dani N, Mahale S. Periodontal vaccine: A dream or reality. J Indian Soc Periodontol. 2011; 15: 115-120.

73. Pouliot M, Clish CB, Petasis NA, Van Dyke TE, Serhan CN, et al. Lipoxin A4 analogues inhibit leukocyte recruitment to Porphyromonasgingivalis, a role for COX- 2 \&lipoxins in periodontal disease. Biochemistry. 2000; 39: 47614768.

74. Serhan CN, Gotlinger K, Hong S, Lu Y, Siegelman J, Baer T, et al. Antiinflammatory actions of neuroprotectin D1/protectin D1 and its natural stereoisomers: assignments of dihydroxy-containing docosatrienes. J Immunol. 2006; 176: 1848-1859.

75. Heijl L, Heden G, Svärdström G, Ostgren A. Enamel matrix derivative (EMDOGAIN) in the treatment of intrabony periodontal defects. J Clin Periodontol. 1997; 24: 705-714.

76. Herpin A, Lelong C, Favrel P. Transforming growth factor-beta-related proteins: an ancestral and widespread superfamily of cytokines in metazoans. Dev Comp Immunol. 2004; 28: 461-485.

77. Reddi AH, Reddi A. Bone morphogenetic proteins (BMPs): from morphogens to metabologens. Cytokine Growth Factor Rev. 2009; 20: 341-342.

78. Van Dyke TE. Control of inflammation and periodontitis. Periodontol 2000 2007; 45: 158-166

79. Hasturk H, Kantarci A, Goguet-Surmenian E, Blackwood A, Andry C, Serhan $\mathrm{CN}$, et al. Resolvin $\mathrm{E} 1$ regulates inflammation at the cellular and tissue leve and restores tissue homeostasis in vivo. J Immunol. 2007; 179: 7021-7029.

80. Bannenberg GL, Chiang N, Ariel A, Arita M, Tjonahen E, Gotlinger KH, et al. Molecular circuits of resolution: formation and actions of resolvins and protectins. J Immunol. 2005; 174: 4345-4355

81. Gilroy DW, Lawrence T, Perretti M, Rossi AG. Inflammatory resolution: new opportunities for drug discovery. Nat Rev Drug Discov. 2004; 3: 401-416. 
82. Murphy FJ, Seery LT, Hayes I. Therapeutic approaches to the modulation of apoptosis. Essays Biochem. 2003; 39: 131-153.
83. Haskó G, Cronstein BN. Adenosine: an endogenous regulator of innate immunity. Trends Immunol. 2004; 25: 33-39.
Austin J Dent - Volume 4 Issue 6 - 2017

ISSN : 2381-9189 | www.austinpublishing group.com

Mohindra et al. () All rights are reserved
Citation: Mohindra K and Nirola A. Host Modulation. Austin J Dent. 2017; 4(6): 1086.

Austin J Dent 4(6): id1086 (2017) - Page - 09 